\title{
Modeling of RFID-Enabled Real-Time Manufacturing Execution System in Mixed-Model Assembly Lines
}

\author{
Zhixin Yang, ${ }^{1}$ Wei Xu, ${ }^{2}$ Pak-Kin Wong, ${ }^{1}$ and Xianbo Wang ${ }^{1}$ \\ ${ }^{1}$ Department of Electromechanical Engineering, Faculty of Science and Technology, University of Macau, Macau \\ ${ }^{2}$ School of Management, Henan University of Science and Technology, Luoyang 471000, China \\ Correspondence should be addressed to Zhixin Yang; zxyang@umac.mo
}

Received 7 May 2014; Revised 9 July 2014; Accepted 14 July 2014

Academic Editor: Qingsong Xu

Copyright (c) 2015 Zhixin Yang et al. This is an open access article distributed under the Creative Commons Attribution License, which permits unrestricted use, distribution, and reproduction in any medium, provided the original work is properly cited.

\begin{abstract}
To quickly respond to the diverse product demands, mixed-model assembly lines are well adopted in discrete manufacturing industries. Besides the complexity in material distribution, mixed-model assembly involves a variety of components, different process plans and fast production changes, which greatly increase the difficulty for agile production management. Aiming at breaking through the bottlenecks in existing production management, a novel RFID-enabled manufacturing execution system (MES), which is featured with real-time and wireless information interaction capability, is proposed to identify various manufacturing objects including WIPs, tools, and operators, etc., and to trace their movements throughout the production processes. However, being subject to the constraints in terms of safety stock, machine assignment, setup, and scheduling requirements, the optimization of RFID-enabled MES model for production planning and scheduling issues is a NP-hard problem. A new heuristical generalized Lagrangian decomposition approach has been proposed for model optimization, which decomposes the model into three subproblems: computation of optimal configuration of RFID senor networks, optimization of production planning subjected to machine setup cost and safety stock constraints, and optimization of scheduling for minimized overtime. RFID signal processing methods that could solve unreliable, redundant, and missing tag events are also described in detail. The model validity is discussed through algorithm analysis and verified through numerical simulation. The proposed design scheme has important reference value for the applications of RFID in multiple manufacturing fields, and also lays a vital research foundation to leverage digital and networked manufacturing system towards intelligence.
\end{abstract}

\section{Introduction}

In recent years, with the development of information technology and advanced manufacturing technology, modern manufacturing industries have changed greatly to match fierce global competition. The development trends include increased personalization, diversified product demands, gradually shortening product life cycle, and increasing delivery requirements from customers, which make shop-floor management be crucial in the whole manufacturing system. To quickly respond to the diverse product demands in terms of multiple product types and different delivery times, mixedmodel assembly lines, as a flexible production mode, are well adopted in discrete manufacturing industries. Compared with single mass production, mixed-model assembly production needs to handle complex material distribution.
Mixed-model assembly involves a variety of parts, complex processes, and fast production changes, which greatly increase the difficulty for agile production management. The workstations used, process sequences, and operating time vary according to different products that are fed into the mixed-model assembly lines. The production management of shop-floor with mixed-model assembly lines faces challenges to reach agility when information among manufacturing objects could not be acquired and processed to generate interactive means in real-time. Although many enterprises have adopted ERP or MRP for leveraging production management, however, in dynamic shop-floor environment, materials can hardly be distributed accurately and sequentially due to the lack of real-time production status tracking. Meanwhile, unpredictable and fast changing market demands require manufacturing enterprises to have quick response 
ability, which, however, is usually insufficient in the currently available production line modeling and simulation software packages. The performance of manufacturing process agility is a major determinant of whether or not enterprises could process manufacturing events quickly, respond to dynamic demands of market customers robustly, build dynamic alliances efficiently with partners for increasing innovative capabilities, and eventually win in global competition. Therefore, aiming at breaking through the development bottlenecks in existing production management, a novel manufacturing execution system (MES), which is featured with real-time and active information interaction technologies, is urgently required to be deployed in mixed-model assembly shop-floor through innovative production organization and management model.

Meanwhile, RFID (Radio Frequency Identification) technology has been increasingly realized as a promising information interaction tool and has been widely used in manufacturing industries to conduct real-time monitoring of large amount of site data. Owing to the intrinsic properties of RFID technology including large information storage capacity, ability to simultaneously identify multiple tags in one moment, and noncontact recognition, it enables a fundamental change in the ways to control industry process, especially in fields of manufacturing and logistics supply chain. Potential benefit and influence of RFID technology on manufacturing industry include increasing the visibility and accuracy of information, real-time production control, improving the flexibility of production planning and scheduling, tracking production related objects (such as Work-In-Process (WIP), workstations, and workers), tracking the utilization rate of reusable assets (such as containers, tools, and removable equipment), effective maintenance management, inventory control of raw materials and finished products, cost reduction, and improving customer satisfaction level $[1,2]$. Therefore, RFID is an attractive technology for fitting real-time requirements in terms of data acquisition and process control in modern manufacturing system. However, existing researches on the application of RFID technology mainly focus on how to build corresponding RFID application systems individually. In the open literature, the theoretical model regarding the optimal configuration and framework integration of RFID sensor networks in various applications are still lacking a unified and detailed study. Many systematic researches are based on the assumption that the acquisition system underlying RFID could meet specific application environment by generating signal in ideal status, while, in fact, RFID system may output unreliable data. Few researchers addressed how to optimize RFID system performance to reduce the influence of noise factors and guarantee system stability in specific application environment. This paper adopts RFID equipment, the high caliber wireless communication tool, for real-time information acquisition and processing in complex production system towards real-time MES. The relevant model configuration, data purifying, and optimization issues are therefore worth being studied in this paper.

The remainder of this paper is organized as follows. In the following section, relevant works related to this study will be reviewed. The system architecture, model analysis for optimization of system configuration, and production planning and scheduling are described in Section 3. In addition, the reliability of RFID data processing and generalized Lagrangian decomposing approach are presented in Sections 4 and 5, respectively. The algorithm analysis and model validity are examined in Section 6. Finally, a summary of modeling methodology, together with a discussion on the directions for future research, is made.

\section{Literature Review}

To study a real-time MES model for complex product manufacturing system, the study involves research efforts in two subareas: wireless technique for information acquisition and optimization of production planning and scheduling. Correspondingly, this section reviews the applications of RFID for real-time information acquisition and facility monitoring in manufacturing execution system and also gives a brief summary on production planning and scheduling optimization studies of manufacturing system.

2.1. RFID-Enabled Real-Time MES. Researches on the application of RFID in manufacturing process control mainly addressed the following three aspects: determination of specific application fields and functions of RFID technology, process control of RFID system, and RFID system integration. Currently, many scholars begin to focus on RFID applications in manufacturing workshops to strengthen production process management by effectively improving the transparency of manufacturing workshop [3-5].

The automatic control problem in production process could be solved using RFID technology [6]. A RFID based intelligent product was proposed to make products have automatic information interaction, accurate information, and real-time acquisition ability and thus support workshop production decisions and automatic control. Based on the characteristic comparison of RFID and barcode technology, RFID was selected to manufacture control system based on programmable logic controller. The control system was combined with multi-agent technology as information interaction media between RFID equipment and other agents [7]. A manufacturing system framework based on RFID was therefore built. Huang's group [8-10] proposed the concept of realizing JIT through wireless manufacturing (WM) technology, where WM collected real-time manufacturing workshop site information by fully relying on wireless devices such as RFID, Auto ID sensor, or wireless information network. The application of RFID in remote monitoring in enterprise production management was reported [11], which could improve the efficiency of production process by readapting IT system and reduce human errors via applying RFID technology. A new device based on RFID technology has been reported to strengthen information exchange among workshops and information system optimization throughout the whole factory [5]. Another effort on system design using RFID could assist grinding apparatus production when combined with ERP system [12]. It was also reported to apply such combined system for logistics control of integrated circuit assembly 
industry in order to improve production efficiency [13]. With respect to production control problem of flexible assembly line, a production control mathematic model based on RFID real-time data collection was built [14], which could be used for designing an intelligent production control decision support system. Chen and Tu [15] put forward a manufacturing control and collaboration system based on multi-agent and applied RFID to real-time manufacturing workshop to monitor and control dynamic production process for improvement of visual tracking ability of product customization of a large number of customers. Risk management system based on RFID technology in manufacturing industry was analyzed, which could not only identify potential risks in production but also propose relevant solutions to control risks [16, 17]. Huang et al. [18] applied WM concept to workshop real-time management of work in process, workshop adaptive assembly planning and control, and fixed worker mobile assembly management of working points. More recently, Huang et al. [19] employed RFID technology and wireless network communication technology (such as Bluetooth and Zigbee) in manufacturing workshop and proposed an advanced wireless manufacturing model via building and manipulating smart workshop objects.

2.2. Production Planning and Scheduling. Production planning and scheduling integrated optimization problem can be solved by three categories of strategies: (1) layered serial solution; (2) layered iteration solution; and (3) full space solution. Production planning and scheduling belong to different decision layers in manufacturing system, respectively. While they are closely related, the decisions made on production planning layer are treated as optimized constraining conditions for the optimization computing in schedule layer, and vice versa. Research works on solving strategies of production planning and scheduling integrated optimization model are summarized in Table 1.

\section{Model Analysis}

3.1. Model Framework. In modern industries, such as automotive and mobile phone makers, mixed-model assembly lines are typical configuration where a set of workpieces will be assigned on different workstations and be transported along correspondingly different assembly lines. This paper assumes that the workshop has $M$ sets of machines with different stamping processing capacity, $\bar{M}=\{1, \ldots, M\} . m$ is machine index and $m \in \bar{M}$. Each machine is installed with a RFID information collection sensor; that is, the RFID readers for relatively fixed manufacturing resources (such as manufacturing equipment) and RFID tags for relatively moving manufacturing resources (such as manufacturing workers, containers for loading materials, and key components and parts) are deployed in the system. When those moving tags that are mounted on manufacturing objects fall into the active identification range of their nearby readers, tags information can be automatically acquired in real-time. Through mapping mechanism, the attached manufacturing resources could then be identified for dynamic control. It is planned to produce $N$ types of workpieces in a period consisting of $T$ production cycles, where $\bar{T}=\{1, \ldots, T\}, \bar{N}=\{1, \ldots, N\}$. In addition, $i, j$ are workpiece indexes, where $i, j=1, \ldots, N$. $v, w$ are product process index, where $v, w=1, \ldots, n_{i}$. Each workpiece $i$ contains $n_{i}$ processes, $\bar{n}_{i}=\left\{1, \ldots, n_{i}\right\} .(i, v)$ is the $v$ th working process of workpiece $i$; we also assume that multiple machines can complete each processing plan simultaneously. In cycle $t(t \in \bar{T})$, the demand of workpiece $i$ is known to be $d_{i, t}$. Reasonable production planning and scheduling are required to be conducted so as to ensure all of the production tasks in each cycle can be completed, and processing time can be extended within appropriate ranger.

The RFID configuration in sensing network for information collection in mixed-model assembly manufacturing is complex. The optimization of such configuration mainly aims at minimizing RFID total costs while meeting the objective of detecting $K$ collection capacity and collection accuracy through $M$ RFID equipment corresponding to manufacturing resources configuration. In addition, the key of production planning is to formulate production capacity $X_{i, t}$ of various workpieces, so as to minimize the total cost of production cost, inventory cost, and shortage penalty cost. Production scheduling is to assign machines for each type of products and to determine product process sequence of each piece of equipment. The start and completion time of each workpiece in one machine could be computed, which may require workers to work overtime in some machines. For minimizing the total cost that includes machine setup cost and overhead, the interacting production planning and scheduling processes will be optimized in an integrated manner that covers all relevant cost while ensuring feasible scheduling.

It is well known that setup process often involves die and mold changes, changes of tools or workpieces and the corresponding calibration, and so forth, which usually take certain significant time during a planning cycle. For the example of stamping process, the stamping part with different setup requirements may be produced insufficiently and thus be unable to meet the demands from the downstream welding workshop. Meanwhile, as product demands are dynamically changed for fitting urgent orders or emergency, welding workshop will increase the demand for stamped parts. To cope with such case, certain level of inventory control method will be explored, typically, how to set the safety stock? Generally, safety stock is a rigid requirement and is treated as a constraint. However, this requirement cannot be met under limited production capacity. Therefore, safety stock can be treated as an objective for fulfilling, instead of a constraint. If the safety stock level cannot be satisfied, penalty cost will be incurred accordingly.

3.2. Notations and Assumption. $I_{i, t}\left(I_{i, t} \geq 0\right)$ is used to denote the inventory of product $i$ at the end of production cycle $t$. If it is greater than the specified safety stock value $R I_{i, t}$, that is, $I_{i, t} \geq R I_{i, t}$, then the exceeded parts are called excess safety stock, which is denoted as $S I_{i, t}^{+}$(i.e., if $I_{i, t} \geq R I_{i, t}$, $\left.S I_{i, t}^{+}=\max \left\{I_{i, t}-R I_{i, t}, 0\right\}\right)$. If the inventory is smaller than $R I_{i, t}, I_{i, t} \leq R I_{i, t}$, then the insufficient part is called shortfall 
TABLE 1: Brief summary of solving strategies for production planning and scheduling optimization.

\begin{tabular}{ll}
\hline Literature works & Research domain and approach \\
\hline$[20,21]$ & $\begin{array}{l}\text { Decomposing according to the structure of integrated model; } \\
\text { iterative standard mathematical programming approach. }\end{array}$ \\
\hline$[22-24]$ & $\begin{array}{l}\text { Decomposing according to the structure of integrated model; } \\
\text { Laplace relaxation decomposition approach. }\end{array}$ \\
\hline$[25-28]$ & $\begin{array}{l}\text { Solve production planning problem under total capacity constraint; } \\
\text { ensure the feasibility of scheduling layer. }\end{array}$ \\
\hline$[29]$ & $\begin{array}{l}\text { Single-stage continuous multiproducts workshop; } \\
\text { simultaneous optimization of production planning and scheduling. }\end{array}$ \\
\hline$[30]$ & $\begin{array}{l}\text { Pharmaceutical industry; layered iterative solution; } \\
\text { multiscale planning and scheduling model. }\end{array}$ \\
\hline$[31]$ & $\begin{array}{l}\text { Production planning and scheduling integrated optimization problem; } \\
\text { standard mathematical programming approach. }\end{array}$ \\
\hline$[32]$ & $\begin{array}{l}\text { Operating workshop; linear programming solution; } \\
\text { production planning and scheduling integrated optimization. }\end{array}$ \\
\hline$[33]$ & $\begin{array}{l}\text { Single-stage production system; linear programming solution; } \\
\text { batch determination and scheduling sequence optimization model. }\end{array}$ \\
\hline
\end{tabular}

safety stock, which is denoted as $S I_{i, t}^{-}$(i.e., if $I_{i, t} \leq R I_{i, t}$, $\left.S I_{i, t}^{-}=\max \left\{R I_{i, t}-I_{i, t}, 0\right\}\right)$. Therefore, the inventory status of one product $i$ is composed of specified safety stock $R I_{i, t}$, safety stock excess $S I_{i, t}^{+}$, and safety stock shortfall $S I_{i, t}^{-}$which could be denoted as $I_{i, t}=R I_{i, t}+S I_{i, t}^{+}-S I_{i, t}^{-}$. The unit penalty costs for safety stock shortfall and excess of product $i$ in production cycle $t$ are represented using variables $C h_{i, t}^{-}, C h_{i, t}^{+}$, respectively, and meet the condition of $\mathrm{Ch}_{i, t}^{-}>\mathrm{Ch}_{i, t}^{+}$. The summary of notations is used in this paper (see Notation).

3.3. Optimal Configuration of RFID Equipment. To leverage wireless application in mixed-model assembly manufacturing environment, identifying physical objects and tracing their movement throughout the production processes will be one of the fundamental supporting factors. Targeted objects are stuck or embedded with RFID tags, which are visible and traceable by those nearby physical readers that are deployed at some key control points. The identified physical objects become intelligent via dynamic information feedback and sharing capability provided by RFID facilities. Each unique RFID tag is mounted with one individual object. When one tag falls into the identification range of the active readers, these active readers will execute simple link layer protocol to retrieve the object identification information embedded in the identified tag. However, due to the characteristics of frequency identification wireless communication and the influence of complex application environment in mixedmodel assembly manufacturing workshop, it suffers from receiving repeated readings of tags, missing reading of tags, and redundant objects data when multiple tags are associated with it. Consequently, the raw RFID data flow is unreliable in terms of data redundancy and incompleteness, which in turn results in difficulties for rational downstream applications and also limits its application feasibility. Therefore, the mixed-model assembly manufacturing environment requires proper configuration of RFID equipment prior to data collection and analysis, which is essential for effective system monitoring. The schematic diagram of RFID based real-time manufacturing information in physical workshop operation system is shown in Figure 1.

The nature of production monitoring techniques is to infer whether the manufacturing objects under tracking are in a specific "state," "event," or matching certain "rules," according to basic object information, such as time, space, and interrelationship. A real-time MES monitoring system requires synthesising all the relevant information related to those selected objects for monitoring and will ignore the other objects and system noise. To sum up, there are lots of objects that can be monitored in any production environment, while it is neither practical nor necessary to implement full monitoring for all of them. For example, the objects under tracking could be selective, and only those high-value parts, or staffs, materials, and equipment located in key regions are included. When designing the model configuration of production monitoring schemes, "cost performance" of monitoring tasks will be adopted as the primary indicator for determination of whether the targeting objects, data collection methods and relevant processing technologies shall be applied or not.

RFID devices are used to track manufacturing objects, by means of reading and writing radio signals, without physical or optical contact with the objects to be identified. The technology mainly consists of two types of sensor mechanisms. Passive sensors are not equipped with internal battery, which absorb and convert energy from RFID reader. Comparably, active sensors send signals by internal power, which can measure multiple types of sensors, such as temperature, pressure, and motion sensors. Which sensor is optimal for the real-time manufacturing process? It mainly depends on the workshop physical operating environment. Active sensor is suitable for tracking moving objects in a wide area, whereas it is a viable choice for passive sensor to identify information in nearby field. In this paper, RFID active and passive tags are assumed to be attached to single component or whole batch 


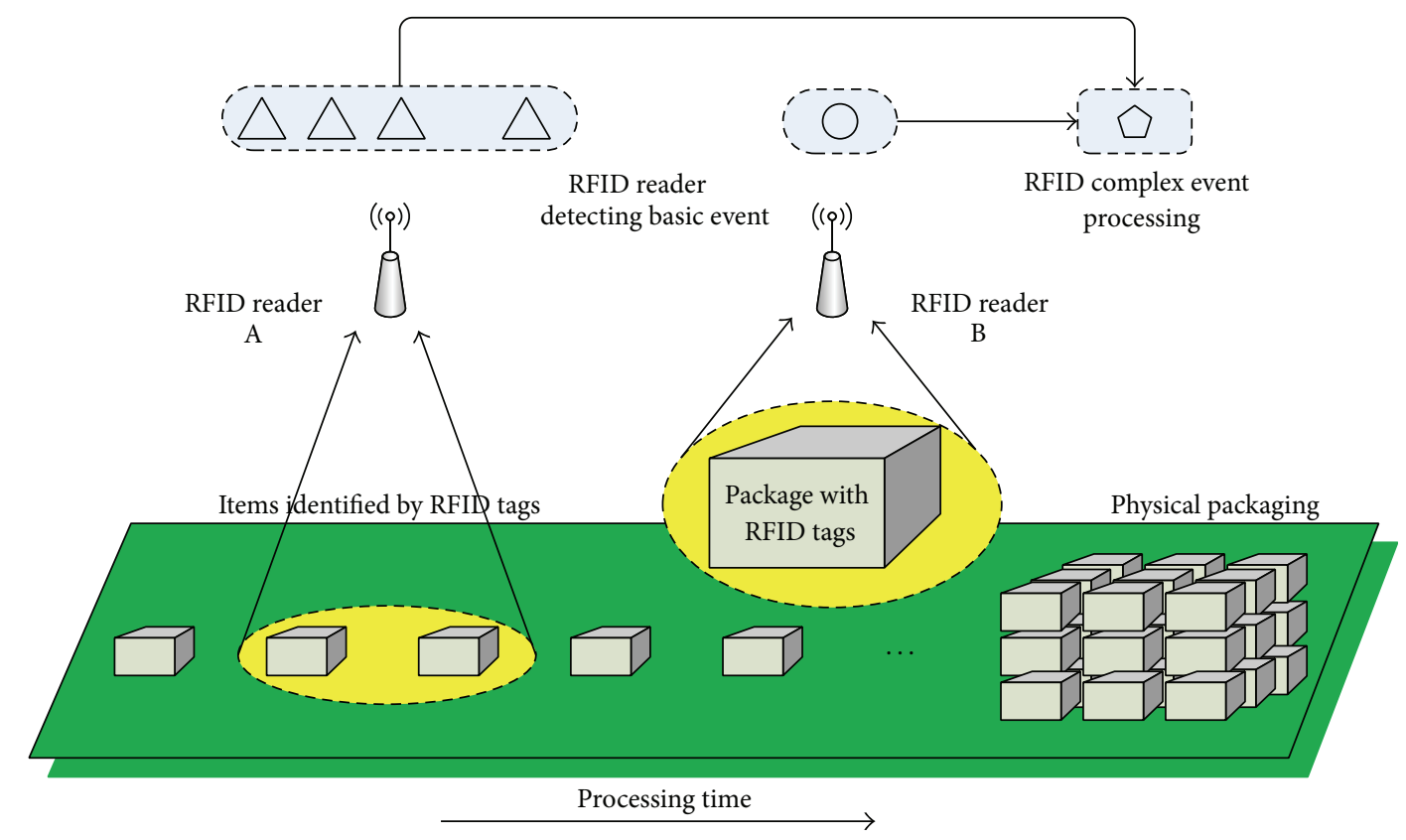

FIGURE 1: Schematic diagram of RFID based real-time MES in physical workshop environment.

package. With respect to RFID equipment configuration regarding sensor network for manufacturing information acquisition in MES, the following mathematical model can be used for computing the optimal configuration:

$$
\begin{aligned}
& \operatorname{Min} Z_{1}=\sum_{i=1}^{M} \operatorname{cost}\left(\text { ActiveSensor } \_i\right) \\
& +\sum_{j=1}^{N} \operatorname{cost}\left(\text { PassiveSensor }_{-} j\right) \text {, } \\
& \left(\chi_{1}, \ldots, \chi_{K}\right) \in\left(\chi_{\text {Sensor } \_1}, \ldots, \chi_{\text {Sensor } \_M+N}\right) \text {, } \\
& \left(\varphi_{1}, \ldots, \varphi_{K}\right) \in\left(\varphi_{\text {Sensor_1 }}, \ldots, \varphi_{\text {Sensor_M+N }}\right) \text {, }
\end{aligned}
$$

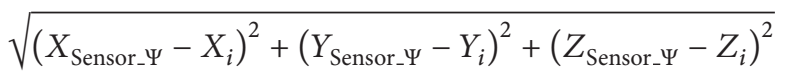

$$
\begin{aligned}
& \leq \Theta_{i}, \\
& \text { Sensor }_{\Psi}= \begin{cases}\text { ActiveSensor }_{-} i(\Psi=i), & i \in[1, M] \\
\text { PassiveSensor }_{-} j(\Psi=j), & j \in[1, N],\end{cases} \\
& \Psi \in[1, M+N],
\end{aligned}
$$

where the objective function is for $K$ number of RFID data acquisitions to be detected, to minimize total costs while meeting the objective of detecting $K$ acquisition quantity and accuracy through $M+N$ sensing equipment (ActiveSensor $i$ and PassiveSensor $-j$ ) and accessory equipment corresponding to manufacturing resource configuration. The constraints are to ensure that the selected sensing equipment could meet $K$ number of RFID acquisitions $\left(\chi_{1}, \ldots, \chi_{K}\right)$ and acquisition accuracy $\left(\varphi_{1}, \ldots, \varphi_{K}\right)$, respectively, as well as to ensure the reliability of sensor equipment configuration; that is, the distances between the installation location of the selected sensing equipment $\left(X_{\text {Sensor_} \Psi}, Y_{\text {Sensor_} \Psi}, Z_{\text {Sensor_} \Psi}\right)$ and working position of effective $\operatorname{Reader}_{i}\left(X_{i}, Y_{i}, Z_{i}\right)$ are within the farthest sensor identification range.

3.4. Optimization of Production Planning. Production planning is for enterprises to make a series of decisions regarding production rate, inventory and shortage quantity, and so forth, for various products under the constraint of production capacity. The optimization of production planning aims at minimizing total expenses of the above decisions, where the production objectives and inventory level in each production cycle form control parameters. Generally, production planning and scheduling are formulated in sequence, that is, generating production planning first and then specifying scheduling based on such planning result. The main drawback of formulating production planning and scheduling by hierarchical approach is that information in detailed scheduling layer is not taken into account when formulating production planning. The resulted scheduling thus tends to be infeasible; that is to say, decisions made according to the planning layer may cause infeasible scheduling. Therefore, machine setup time, which is used for adjusting the machine configuration according to mixed-model assembly workshops processes, will be estimated for production batch optimization. In this paper, machine setup is set as variable $\alpha_{i, v}^{m, t}$; the production planning model could be determined using the formula where the minimum overall production expense is adopted as the objective function:

$$
\begin{gathered}
\operatorname{Min} Z_{2}=\sum_{t=1}^{T} \sum_{i=1}^{N}\left(C h_{i, t}^{+} S I_{i, t}^{+}+C h_{i, t}^{-} S I_{i, t}^{-}+C p_{i, t} X_{i, t}\right. \\
\left.+C b_{i, t} L_{i, t}+C u_{i, t} y_{i, t}\right) .
\end{gathered}
$$


The main expense in this model is composed of penalty cost of safety stock excess, penalty cost of safety stock shortfall, production expenses, shortage cost, and production preparation cost. The constraint conditions are given by

$$
\begin{gathered}
\mathrm{INV}_{i}+L_{i, 1}+X_{i, 1}=I_{i, 1}+d_{i, 1}, \quad \forall i, \\
I_{i, t-1}+L_{i, t}+X_{i, t}=I_{i, t}+d_{i, t}, \quad t=2, \ldots, T, \quad \forall i \\
\sum_{(i, v) \in J(m)}\left(s_{i, v}^{m} y_{i, t}+p_{i, v}^{m} X_{i}, t\right) \alpha_{i, v}^{m, t} \leq \omega^{\prime} W_{t}, \quad \forall m, t \\
S I_{i, t}^{+}=\max \left\{I_{i, t}-R I_{i, t}, 0\right\}, \quad \forall i, t, \\
S I_{i, t}^{-}=\max \left\{R I_{i, t}-I_{i, t}, 0\right\}, \quad \forall i, t, \\
m l_{i} y_{i, t} \leq X_{i, t} \leq M l_{i} y_{i, t}, \quad \forall i, t, \\
L_{i, t} \leq d_{i, t}, \quad \forall i, t, \\
S I_{i, t}^{+}, S I_{i, t}^{-}, I_{i, t}, L_{i, t}, X_{i, t} \in z_{N \times T}^{+}, \quad y_{i, t} \in\{0,1\}, \quad \forall i, t
\end{gathered}
$$

where constraints (3) and (4) express materials balance equations. Formula (5) is production capacity constraint, where $\alpha_{i, v}^{m, t}$ is a constant. $\omega^{\prime}$ represents coefficient of production capacity, and $\omega^{\prime}=1$ if it is not allowed to work overtime; otherwise, $\omega^{\prime}>1$. Constraints (6) and (7) give formulas to calculate safety stock excess and safety stock shortfall, respectively. Constraint (8) represents upper limit and lower limit of production batch. Constraints (9) and (10) restrict the upper limits of safety stock shortfall and workpiece shortage quantity.

3.5. Optimization of Production Scheduling. Production planning and scheduling, which are the two most important tasks in manufacturing production and operation management, are closely related to each other and significantly influence profits gaining, resource utilization efficiency, and punctual product delivery. Solutions of production planning (production objectives) are the input conditions for scheduling problems. Meanwhile, production capacity constraints involved in production planning problems are often related to scheduling solutions.

The main task of production scheduling is to make machines assignment decision and to specify the sequence of production processes in each production cycle formulated in production planning. It could thus determine the start and completion time of each product in every working procedure on corresponding machines. Currently, the key that seriously hinders classical scheduling theoretical researches from making major progress and breakthroughs is NP nature of scheduling problem. The actual scheduling problems are always very complex without certain rules to follow and exact solutions are difficult to be obtained within limited time. Applying the classical scheduling theory based analytic optimization to actual scheduling problems that belong to NP-hard problems will inevitably encounter such obstacles.

The scheduling problem we are handling is with delivery constraint, which assigns machines based on the minimum processing time for each process and machine load condition according to processing schedule from top to bottom. Since such NP-hard scheduling problem cannot find an overall exact solution directly, we propose to partially fix the solved batch planning result as a basis and use it to solve scheduling problem. Such method could finally get a near optimal solution through limited iterations. When the value of production batch $X_{i, t}$ is known, variables $X_{i, t}, y_{i, t}$ in the following constraints are known. Then, production scheduling model is as follows:

$$
\operatorname{Min} Z_{3}=\sum_{m=1}^{M} \sum_{t=1}^{T} \sum_{i=1}^{N} \sum_{v=1}^{n_{i}} C s_{i, v}^{m} \alpha_{i, v}^{m, t}+\sum_{m=1}^{M} \sum_{t=1}^{T} C t_{m, t} O_{m, t} .
$$

Formula (11) takes minimum setup cost in terms of die and mold change, calibration cost, and overtime cost as objective functions. The corresponding constraint conditions are given by

$$
\begin{gathered}
\alpha_{i, v}^{m, t} \leq f_{i, v}^{m}, \quad v \in \bar{n}_{i}, \forall i, m, t \\
C_{i, v}^{t} \geq B_{i, v}^{t}+\sum_{m \in M(i, v)}\left(s_{i, v}^{m} y_{i, t}+p_{i, v}^{m} x_{i}, t\right) \alpha_{i, v}^{m, t}, \quad v \in \bar{n}_{i}, \quad \forall i, t \\
C_{i, v}^{t} \leq B_{i, v+1}^{t}, \quad v \in \bar{n}_{i}, \quad \forall i, t, \\
B_{i, v}^{t}+\left(s_{i, v}^{m} y_{i, t}+p_{i, v}^{m} X_{i, t}\right) \alpha_{i, v}^{m, t}-\left(1-\delta_{i, v, j, w}^{m, t}\right) G \leq B_{j, w}^{t} \\
v \in \bar{n}_{i}, \quad w \in \bar{n}_{j}, \quad \forall i, j, m, t \\
B_{j, w}^{t}+\left(s_{j, w}^{m} y_{j, t}+p_{j, w}^{m} x_{j, t}\right) \alpha_{j, w}^{m, t}-\left(1-\delta_{j, w, i, v}^{m, t}\right) G \leq B_{i, v}^{t} \\
v \in \bar{n}_{i}, \quad w \in \bar{n}_{j}, \quad \forall i, j, m, t
\end{gathered}
$$

$$
\sum_{m \in M(i, v)} \alpha_{i, v}^{m, t}=y_{i, t}, \quad v \in \bar{n}_{i}, \forall i, t
$$

$\sum_{(i, v) \in J 1(m)} \delta_{i, v, j, w}^{m, t}=\alpha_{j, w}^{m, t}, \quad m \in M(j, w), w \in \bar{n}_{j}, \forall j, t$

$$
\sum_{(j, w) \in J 2(m)} \delta_{i, v, j, w}^{m, t}=\alpha_{i, v}^{m, t}, \quad m \in M(i, v), v \in \bar{n}_{i}, \forall i, t,
$$

$$
\sum_{(i, v) \in J(m)} \delta_{0, w, i, v}^{m, t} \leq 1, \quad w=m, \forall m, t
$$$$
\sum_{(i, v) \in J 2(m)} \delta_{i, v, 0, w}^{m, t}=0, \quad w=m, \forall m, t,
$$$$
\sum_{(i, v) \in J(m)} \delta_{i, v, n+1, w}^{m, t} \leq 1, \quad w=m, \forall m, t,
$$

$$
\sum_{(i, v) \in J 1(m)} \delta_{n+1, w, i, v}^{m, t}=0, \quad w=m, \forall m, t
$$

$$
F_{m, t}=\max _{\left(i, n_{i}\right) \in J(m)}\left\{C_{i, n_{i}}^{t}-\left(1-\delta_{i, n_{i}, n+1, l}^{m, t}\right) G\right\}, \quad w=m, \forall m, t,
$$

$$
\begin{gathered}
F_{m, t} \leq W_{t}+0_{m, t}, \quad \forall m, t, v, \\
0 \leq 0_{m, t} \leq \omega W_{t}, \quad \forall m, t
\end{gathered}
$$




$$
\begin{aligned}
& B_{i, v}^{t}, C_{i, v}^{t}, 0_{m, t} \geq 0, \quad \alpha_{i, v}^{m, t}, \delta_{i, v, j, w}^{m, t} \in\{0,1\}, \\
& 0 \leq \omega \leq 1, \quad v \in \bar{n}_{i}, \quad w \in \bar{n}_{j}, \quad \forall i, j, m, t
\end{aligned}
$$

where constraint (12) expresses that working procedure can only be processed on the allowed machines. Formulas (13) and (14) are processing sequence constraints of the same workpiece, respectively. Constraints (15) and (16) mean production adjustment and processing work, which can only complete at most one working procedure at the same time, on any one machine in the same period, respectively. Constraint (17) shows that each process can only be processed on one machine when producing products. Constraints (18) and (19) emphasize that each working procedure has one immediate predecessor activity and one immediate successor activity. Constraints (20)-(23) denote that virtual working procedure can only be processed firstly and lastly on each machine. Constraint (24) gives an approach to calculate completion time of each machine. Formula (25) indicates that completion time of each machine cannot exceed the available time, which ensures feasibility of scheduling, and constraint (26) remarks the overtime limit.

\section{Reliability of RFID Data Processing}

The ability to track real-time manufacturing information of workshop physical operating environment is the key for achieving agile production management. RFID technology is a promising technology to acquire real-time manufacturing feedback information. However, data acquisition using RFID technology in complex manufacturing environment may encounter redundant or missing readings, and signal fluctuation due to obstacles and noise. Consequently, a large amount of unreliable data flow will be output to the downstream MES applications. Therefore, how to process RFID events so as to provide reliable and meaningful real application information for downstream applications is the key technical issue that will be solved for successful application of RFID.

This paper proposes a hierarchical data processing model to improve RFID system application reliability in multitag and multireader mixed-model manufacturing system application environment. It could clean and compensate unreliable data and solve RFID abnormal reading phenomena from the perspective of system application, so as to improve system identification rate and reliability level. Data processing model uses a hierarchical structure, which is composed of RFID equipment network layer, basic event processing layer, complex event processing layer, and application layer, as shown in Figure 2. RFID equipment network generates basic events using a set of multiple types of readers that receive signal from neighbouring tags. Redundant RFID basic events caused by repeated reading could be filtered out and form simplified logical reading events. Complex event processing layer receives logical reading events uploaded by basic event processing layer. It could analyze and classify logical reading events based on default application integrity constraint rules, then be able to detect abnormal reading, and perform cleanup operations on received unreliable data. Such data process module will ensure the system integrity, provide meaningful application data, and output reliable interrelated information among tags and their associated objects, such as interdistance and moving directions, for agile production management applications in the application layer.

4.1. RFID Basic Event Processing. Plenty of data recording signals fired by tags will be sent out by the distributed logical control point reader network as input for the basic event processing layer. The distributed deployment in logical control points and its structure consists of distributed physical reader adapter, RFID basic event queue, tag event filter, logical reading event filter, object event queue, and logical mapping engine, as shown in Figure 3. The logical mapping engine stores the mapping relations between one identified tag and its corresponding physical object.

Reader adapter first collects raw data generated by corresponding physical readers and forms RFID basic events for uploading into tag event filter, which cleans redundant tag information collected by multiple readers according to the unique tag logical matching mechanism.

In multitag and multireader application schemes, more than one tag event in RFID basic event queue may be pointed to one physical object. In other words, RFID basic events may contain repeated records for single physical object. Logical reading event filter will filter out duplicated object events according to logical mapping engine via following the principle of not allowing repeated physical object event in each logical control point. Finally, each object and its solely associated tag event are stored in the object event queue and will be uploaded into the correlated logical control point for further processing in the complex event processing module.

4.2. RFID Complex Event Processing. Although basic event layer could clean repeated and redundant data in logical control points and eliminate duplicated tag reading by multiple readers, it does not deal with unreliability caused by redundant and missing reading of tags. Complex event processing layer will be followed to improve the reliability of RFID system application through detecting and correcting redundant data according to RFID application integrity constraint rules. Integrity constraints are based on different perspectives, such as weight, location and motion paths of tag, and, of course, their associative identified physical objects. The constraints could also be based on mutual relations among different objects such as inclusion and exclusion relationships. The structure of complex event processing layer is shown in Figure 4, which mainly consists of manufacturing object information base, integrity constraint rules base, event classification engine, normal event processor, redundant reading processor, and missing reading processor. Integrity constraint rules are the fundamental rules and are set as the default constraint in initial condition.

In manufacturing system, some identified physical objects tend to move along predetermined routes to destinations, such as WIP in production lines and warehouse products. When the detected actual route is different from the pre-specified one, it can report that physical object has encountered unreliable reading phenomena at some 


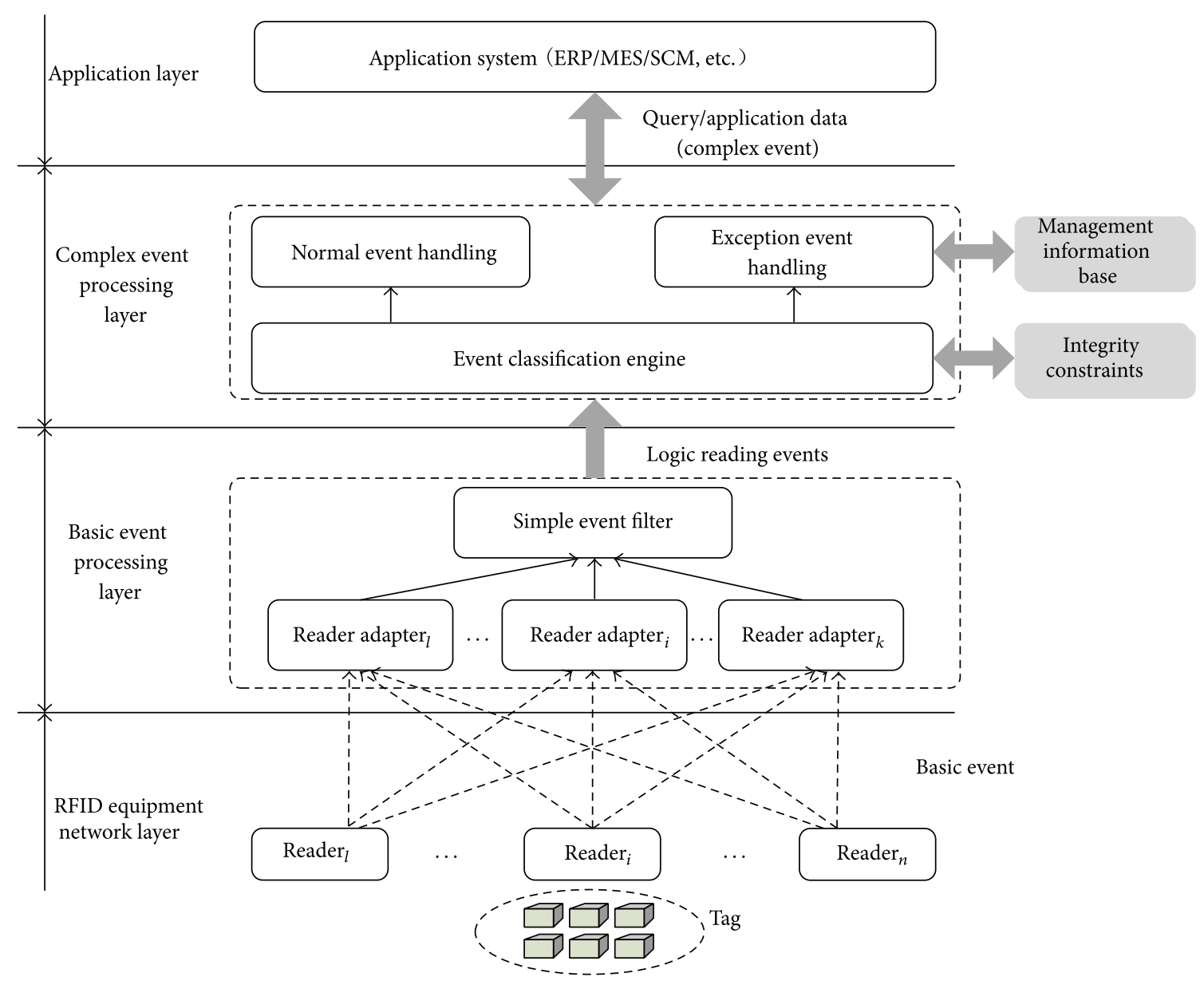

FIGURE 2: Data processing model structure of RFID manufacturing information system reliability.

logical control points. So, redundant and missed read-write phenomena are extracted using management information base that records motion routes of physical objects. The associated relationship between objects and schema is also utilized.

\section{Generalized Lagrangian Decomposition Approach}

The model described above contains RFID optimal configuration, production planning, and scheduling optimization, which belongs to a typical NP-hard problem. Although no exact solution could be found within the limited time, this paper proposes to solve the problem using a novel Lagrangian decomposition approach. By introducing three groups of new constraints and setting $\chi(z)=1$, if $z>0$, and $\chi(z)=0$, otherwise; the overall objective function can be shown as follows:

$$
\operatorname{Min} Z=\operatorname{Min} Z_{1}+\operatorname{Min} Z_{2}+\operatorname{Min} Z_{3}
$$

The new constraints for integrated optimization model include the following:

$$
X_{i, t}=Q_{i, t}, \quad \forall i, t,
$$

$$
\begin{gathered}
\alpha_{i, v}^{m, t}=\beta_{i, v}^{m, t}, \quad \forall i, t \\
\bar{y}_{i, t}=\chi\left(Q_{i, t}\right), \quad \forall i, t \\
\sum_{(i, v) \in J(m)}\left(s_{i, v}^{m} y_{i, t}+p_{i, v}^{m} x_{i}, t\right) \beta_{i, v}^{m, t} \leq W_{t}+O_{m, t}, \quad \forall m, t \\
C_{i, v}^{t} \geq B_{i, v}^{t}+\sum_{m \in M(i, v)}\left(s_{i, v}^{m} \bar{y}_{i, t}+p_{i, v}^{m} Q_{i, t}\right) \alpha_{i, v}^{m, t}, \quad v \in \bar{n}_{i}, \quad \forall i, t \\
C_{i, v}^{t} \geq B_{i, v}^{t}+\sum_{m \in M(i, v)}\left(s_{i, v}^{m} y_{i, t}+p_{i, v}^{m} X_{i, t}\right) \beta_{i, v}^{m, t}, \quad v \in \bar{n}_{i}, \quad \forall i, t \\
B_{i, v}^{t}+\left(s_{i, v}^{m} \bar{y}_{i, t}+p_{i, v}^{m} Q_{i, t}\right) \alpha_{i, v}^{m, t}-\left(1-\delta_{i, v, j, w}^{m, t}\right) G \leq B_{j, w}^{t}, \\
v \in \bar{n}_{i}, \quad w \in \bar{n}_{j}, \quad \forall i, j, m, t \\
B_{j, w}^{t}+\left(s_{j, w}^{m} \bar{y}_{j, t}+p_{j, w}^{m} Q_{j, t}\right) \alpha_{j, w}^{m, t}-\left(1-\delta_{j, w, i, v}^{m, t}\right) G \leq B_{i, v}^{t}, \\
v \in \bar{n}_{i}, \quad w \in \bar{n}_{j}, \quad \forall i, j, m, t
\end{gathered}
$$

$$
\sum_{m \in M(i, v)} \alpha_{i, v}^{m, t}=\bar{y}_{i, t}, \quad v \in \bar{n}_{i}, \forall i, t
$$




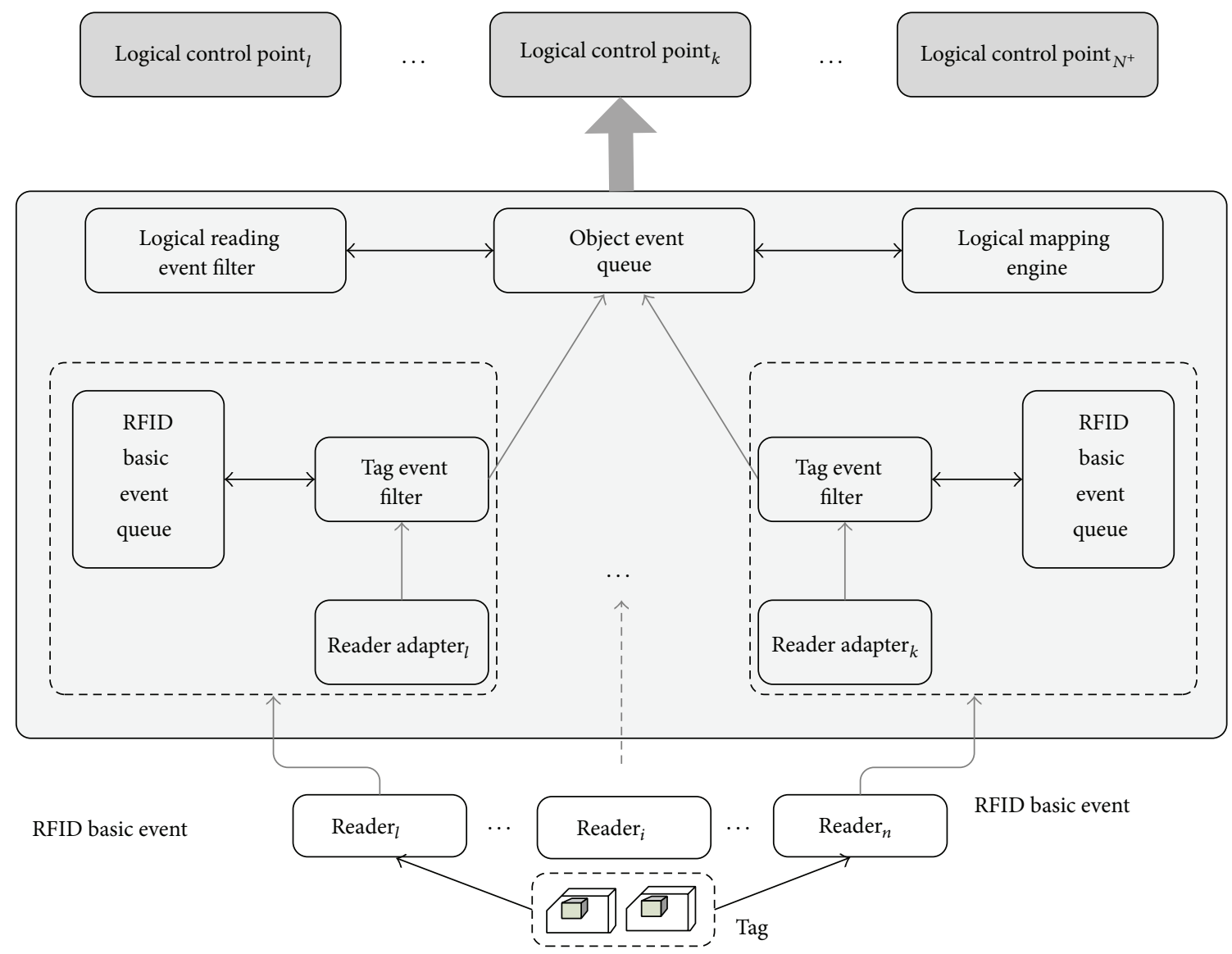

FIGURE 3: Structure of RFID basic event processing layer.

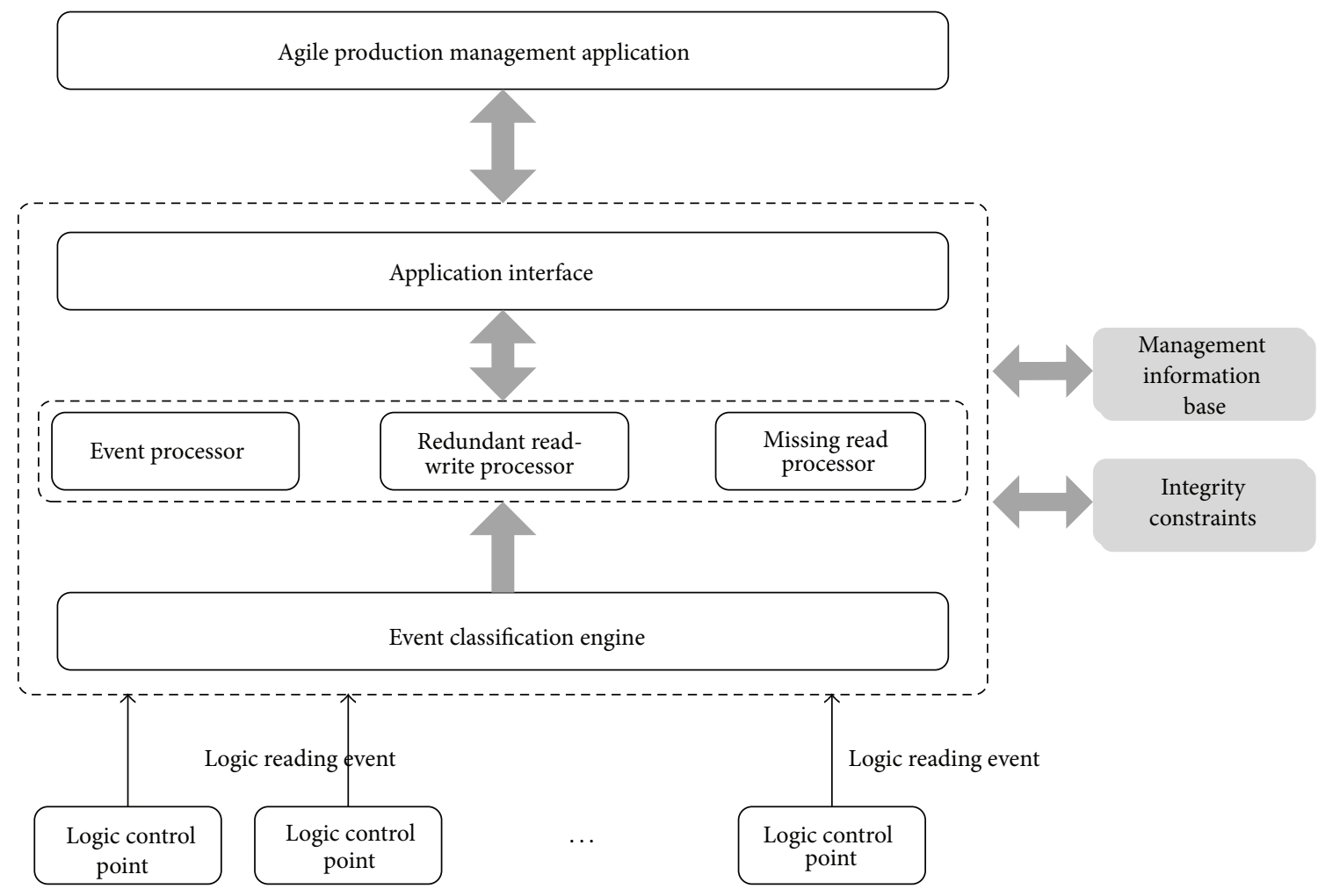

FIGURE 4: Structure of RFID complex event processing layer. 
where $Q_{i, t}, \beta_{i, v}^{m, t}$, as specified in constraints (29) and (30), are artificial variables that connect production planning and scheduling. Constraint (31) determines whether product $i$ is produced in cycle $t$ and ensures that production preparation is carried out only when certain kinds of products are determined to be processed. In order to use Lagrangian algorithm, which carries out computing the variables in production planning and scheduling will be transferred and coordinated in each time of Lagrangian iteration cycle. The variables $Q_{i, t}, \bar{y}_{i, t}$ in scheduling problem are set to be the corresponding output values $X_{i, t}, y_{i, t}$, computed in the production planning process. Similarly, variable $\alpha_{i, v}^{m, t}$ of machine setup in scheduling problem is assigned as $\beta_{i}^{m}$ used in planning problem. In order to decompose and coordinate planning and scheduling, constraints (32) and (34) are followed in Lagrangian treatment of planning subproblem, where $\beta_{i, v}^{m, t}$ is a constant.

Formulas (33), (35), and (36) will be used in scheduling subproblem of Lagrangian approach $\left(Q_{i, t}\right.$ and $\bar{y}_{i, t}$ are treated as constants). Constraint (33) shows that processing machines are assigned only when there is production demand, that is, $\bar{y}_{i, t}=1$. We introduce Lagrangian multipliers $\mu=\left\{\mu_{i, t}\right\}, v=\left\{v_{i, v}^{m, t}\right\}, i \in \bar{N}, v \in \bar{n}_{i}, m \in \bar{M}, t \in \bar{T}$, and $\theta=(\mu, v)$. Lagrangian multipliers $\mu_{i, t}, v_{i, v}^{m, t}$ correspond to the artificial variables $Q_{i, t}$ and $\beta_{i}^{m}$, which will be considered as shadow prices. $\mu_{i, t}$ can be interpreted as profits obtained by selling unit production capacity and resources, and $v_{i, v}^{m, t}$ is profits gained by enterprises leasing equipment.

Lagrangian multipliers $\mu=\left\{\mu_{i, t}\right\}, v=\left\{v_{i, v}^{m, t}\right\}$ are used in the objective function to relax the constraints; the corresponding relaxation model is then given by

$$
\begin{aligned}
Z_{\mathrm{LR}}= & Z+\sum_{i=1}^{N} \sum_{t=1}^{T} \mu_{i, t}\left(X_{i, t}-Q_{i, t}\right) \\
& +\sum_{t=1}^{T} \sum_{m=1}^{M} \sum_{i=1}^{N} \sum_{v=1}^{n_{i}} v_{i, v}^{m, t}\left(\alpha_{i, v}^{m, t}-\beta_{i, v}^{m, t}\right) \\
= & \sum_{t=1}^{T} \sum_{i=1}^{N}\left(C h_{i}^{+} S I_{i, t}^{+}+C h_{i}^{-} S I_{i, t}^{-}+\left(C p_{i, t}+\mu_{i, t}\right) X_{i, t}\right. \\
& \left.+C b_{i, t} L_{i, t}+C u_{i, t} y_{i, t}\right) \\
& -\sum_{t=1}^{T} \sum_{m=1}^{M} \sum_{i=1}^{N} \sum_{v=1}^{n_{i}} v_{i, v}^{m, t} \\
& +\sum_{t=1}^{T} \sum_{m=1}^{M} \sum_{i=1}^{N} \sum_{v=1}^{n_{i}}\left(C s_{i, v}^{m}+v_{i, v}^{m, t}\right) \alpha_{i, v}^{m, t} \\
& +\sum_{m=1}^{M} \sum_{t=1}^{T} C t_{m, t} O_{m, t}, \\
& \mu_{i, t}=\lambda C p_{i}, \quad v_{i, v}^{m, t}=\lambda C s_{i, v}^{m}, \quad 0 \leq \lambda \leq 1 .
\end{aligned}
$$

Note that this model in fact cannot be decomposed into mutually independent subproblems of planning and scheduling, and thus this problem cannot be solved by being decomposed into a series of subproblems by standard Lagrangian decomposition approach. However, approximate solution of this problem can be obtained by a generalized Lagrangian decomposition approach. That is to say, this problem is decomposed into two subproblems, involving production plan problem assigned by fixed machines and production scheduling problem with fixed volume, and then is solved by alternating iterations. This generalized Lagrangian decomposition approach can be seen as a combination of standard Lagrangian approach with Gauss-Seidel iterative principle. In previous literature, the established aggregate production planning model is decomposed into labor model and production planning model, when studying production batch planning problem of labor-constrained production system. However, these two subproblems are not independent, and production planning model involves two variables, namely, production batch and labor force, among which quantity variable of labor is related to variables in labor subproblem. Besides, when solving inventory routing problem by Lagrangian, this problem is decomposed into inventory problem and routing problem. Vehicle distribution route is fixed when solving inventory problem, while inventory variable is seen as a constant when optimizing distribution vehicle routes. Therefore, these are essentially generalized Lagrangian decomposition approach.

We can set $x=\left(S I^{+}, S I^{-}, X, L, y\right)$ and $\pi=(\alpha, \delta, B, C)$ as decision variables of batch production plan and scheduling subproblem, respectively, and denote $\bar{Z}_{2}(x \mid \theta, \bar{\beta})$ as relaxation production planning problem with fixed machine assignment $\beta$ as $\bar{\beta}$ and $\bar{Z}_{3}(\pi \mid \theta, \bar{Q})$ as relaxation scheduling subproblem with fixed production batch $Q$ as $\bar{Q}$. Machine assignment variable $\beta_{i, v}^{m, t}$ can be fixed as constant $\bar{\beta}_{i, v}^{m, t}$. In each time of iteration of Lagrangian algorithm, take the value of constant $\bar{\beta}_{i, v}^{m, t}$ in production planning as constant $\bar{\delta}_{i, v, j, w}^{m, t}$; that is, fix the sequencing results of scheduling subproblem in last iteration and substitute $\bar{\beta}_{i, v}^{m, t}$ and $\bar{\delta}_{i, v, j, w}^{m, t}$ into original constraint formula. Thus, we can get Lagrangian model of production planning subproblem specifically as follows:

$$
\begin{aligned}
& \bar{Z}_{2}(x \mid \theta, \bar{\beta}) \\
&=\sum_{t=1}^{T} \sum_{i=1}^{N}\left(C h_{i}^{+} S I_{i, t}^{+}+C h_{i}^{-} S I_{i, t}^{-}\right. \\
&\left.\quad+\left(C p_{i}, t+\mu_{i, t}\right) X_{i, t}+G b_{i, t} L_{i, t}+C u_{i, t} y_{i, t}\right) \\
& \quad-\sum_{t=1}^{T} \sum_{m=1}^{M} \sum_{i=1}^{N} \sum_{v=1}^{n_{i}} v_{i, v}^{m, t} \bar{\beta}_{i, v}^{m, t}, \\
& \sum_{(i, v) \in J(m)}\left(s_{i, v}^{m} y_{i, t}+p_{i, v}^{m} x_{i, t}\right) \bar{\beta}_{i, v}^{m} \leq(1+\omega) W_{t}, \quad \forall m, t, \\
& C_{i, v}^{t} \geq B_{i, v}^{t}+m \sum_{m \in M(i, v)}\left(s_{i, v}^{m} y_{i, t}+p_{i, v}^{m} x_{i, t}\right) \bar{\beta}_{i, v}^{m}, \quad \forall m, t,
\end{aligned}
$$




$$
\begin{gathered}
B_{i, v}^{t}+\left(s_{i, v}^{m} y_{i, t}+p_{i, v}^{m} X_{i, t}\right) \bar{\beta}_{i, v}^{m, t}-\left(1-\bar{\delta}_{i, v, j, w}^{m, t}\right) G \leq B_{j, w}^{t}, \\
v \in \bar{n}_{i}, \quad w \in \bar{n}_{j}, \quad \forall i, j, m, t, \\
B_{j, w}^{t}+\left(s_{j, w}^{m} y_{j, t}+p_{j, w}^{m} X_{j, t}\right) \bar{\beta}_{j, w}^{m, t}-\left(1-\bar{\delta}_{j, w, i, v}^{m, t}\right) G \leq B_{i, v}^{t}, \\
v \in \bar{n}_{i}, \quad w \in \bar{n}_{j}, \quad \forall i, j, m, t \\
F_{m, t}=\max _{\left(i, n_{i}\right) \in J(m)}\left\{C_{i, n_{i}}^{t}-\left(1-\bar{\delta}_{i, n_{i}, n+1, w}^{m, t}\right) G\right\}, \\
w=m, \quad m \in \bar{M}, \quad t \in \bar{T} .
\end{gathered}
$$

Formula (41) is production capacity constraint with fixed machine assignment variable, and the remaining formulas are production batch constraints after fixing machine processing sequence, aiming at completing formulated production planning in prescribed production cycle, that is, ensuring that the obtained planning is feasible. It is worth pointing out that, for overtime variable $O_{m}$ of each cycle in Lagrangian production planning model, $t$ is substituted by the maximum available overtime, and this change does not affect solution to the objective function, because in the optimal solution, the batch planning ensures obtaining feasible scheduling, while specific overtime is determined by Lagrangian scheduling subproblem. Here, we denote variable $Q_{i, t}$ in the above constraints as constant $\bar{Q}_{i, t}$ and reach constraints of Lagrangian scheduling subproblem. During the running of Lagrangian algorithm, for each time of iteration, $\bar{Q}_{i, t}$ takes batch value $X_{i, t}$ obtained in last iteration in production planning. Lagrangian scheduling model is specifically given by

$$
\begin{gathered}
\bar{Z}_{3}(\pi \mid \theta, \bar{Q}) \\
=\sum_{m=1}^{M} \sum_{t=1}^{T} C t_{m, t} \sum_{t=1}^{T} \sum_{m=1}^{M} \sum_{i=1}^{N} \sum_{v=1}^{n_{i}}\left(C s_{i, v}^{m}+v_{i, v}^{m, t}\right) \alpha_{i, v}^{m, t} \\
-\sum_{i=1}^{N} \sum_{t=1}^{T} \mu_{i, t} \bar{Q}_{i, t}, \\
\bar{y}_{i, t}=x\left(\bar{Q}_{i, t}\right), \quad \forall, i, t, \\
C_{i, v}^{t} \geq B_{i, v}^{t}+\sum_{m \in M(i, v)}\left(s_{i, v}^{m} \bar{y}_{i, v}+p_{i, v}^{m} \bar{Q}_{i, v}\right) \alpha_{i, v}^{m}, \quad v \in \bar{n}_{i}, \quad \forall i, t \\
B_{i, v}^{t}+\left(s_{i, v}^{m} \bar{y}_{i, v}+p_{i, v}^{m} \bar{Q}_{i, v}\right) \alpha_{i, v}^{m, t}-\left(1-\delta_{j, w, i, v}^{m, t}\right) G \leq B_{i, v}^{t}, \\
v \in \bar{n}_{i}, \quad w \in \bar{n}_{j}, \quad \forall i, j, m, t .
\end{gathered}
$$

With a given batch planning, Lagrangian relaxation scheduling problem (LRSP) is a scheduling problem with delivery constraint and is solved by hierarchical approach in this paper. Firstly, an improved localization approach is designed to determine machine assignment problem and assigns machines for operating process with machine load conditions. The main idea is to form a processing schedule firstly by lining up processes of all workpieces (the same workpieces are not separated when lining up, and workpieces are lined up according to workpiece number) and then assign machines based on the minimum processing time of each process and machine load conditions according to processing schedule from top to bottom. However, the assignment results rely on workpiece arrangement locations. References [34, 35] improve this method, that is, selecting two workpieces randomly and exchanging locations of both workpieces in the schedule. This paper makes further improvement; that is, machines are not required to be assigned for processes in strict accordance with sequence specified in schedule. An unassigned process could be randomly selected each time (select from processes that have not been assigned with machines and have the minimum number of processes) and be assigned with a machine for it according to the minimum processing time and machine load conditions.

\section{Algorithms and Numerical Simulations}

\subsection{RFID Data Processing Algorithm in MES}

Definition 1. At time $\tau$, all tags within the identification range of physical readers are detected, and data interaction is conducted once. That is to say, physical readers detect the tags that generate just one RFID basic event $e$ at time $\tau$. Generally, data generated by RFID basic event can be expressed by triple $p e=(p r, e p c, \tau)$, where $p r$ is a physical reader identifier, $e p c$ is a tag identifier, $\tau$ is the time of event, and pe denotes an example of RFID basic event.

The above definition is used for Algorithm 1.

In consideration of physical object route constraint $R(L P, A)$, where logical control points are corresponding to $l p \in L P$, possible routes of physical object among logical control points are corresponding to $a \in A$. Physical object moves along the prespecified routes $\left\langle l_{p_{1}}, \ldots, l_{p_{m}}\right\rangle$, which are called default object routes. To judge sequence relationship of logical control points on object routes, the following definition is given.

Definition 2. For any two logical control points $l p_{i}$ and $l p_{j}$, if $l p_{i}$ is in front of $l p_{j}$, then physical object passes $l p_{i}$ first and then $l p_{j}$, and $l p_{i}<l p_{j}$. If logical reading event $l e_{k}=\left(l p_{k}, o_{k}, \tau\right)$ is currently received, redundant reading and reading leak phenomena of physical objects can be determined by the following method.

Search for physical object that will pass in default routes in logical control point, $l p_{\text {pre }}$. If $l p_{\text {pre }}=l p_{k}$, physical object is identified in normal status; if $l p_{\text {pre }}<l_{p_{k}}$, it is redundant reading phenomenon; otherwise if $l_{p \text { pre }}>l p_{k}$, physical object has reading leak phenomenon at all logical control points. $l p_{\text {leak }}$ will be positioned in between $l_{p_{k}}$ and $l_{p_{\text {pre }}}$ in default routes $\left\langle l p_{1}, \ldots, l p_{k}, \ldots, l p_{\text {pre }}, \ldots, l p_{j}\right\rangle$. The relationship $l p_{k}<$ $l p_{\text {leak }}<l p_{\text {pre }}$ or $l p_{k}=l p_{\text {leak }}$ will be held.

According to the above definition, RFID complex event processing algorithm can be obtained as in Algorithm 2. 
Step 1. Receive RFID basic reading events $p e_{1}=\left(p r_{1}, e p c_{1}, \tau\right)$ generated by physical readers.

Step 2. Query RFID basic event queue based on $e p c_{1}$, and skip to Step 7 if there are RFID basic events with the same tag numbers.

Step 3. Insert epc into the RFID basic event queue.

Step 4. Search physical object numbers in mapping relation table between objects and tags based on $e p c_{1}$; if none is found, skip to Step 7.

Step 5. Search logical reading events with the same object numbers in logical reading event queue according to physical object numbers. If it is positive, skip to Step 7.

Step 6. Assemble $p e_{1}$ into logical reading events according to physical object numbers, and insert $l e_{k}=\left(l p_{k}, o_{k}, \tau\right)$ into logical reading event queue.

Step 7. Abandon RFID basic event; skip to Step 1.

Algorithm 1: RFID basic event processing algorithm.

Step 1. Receive logical reading event $l e_{k}=\left(l p_{k}, o_{k}, \tau\right)$ uploaded by logical control points where $k$ is from 1 to $K$.

Step 2. Get logical control point $l p_{\text {pre }}$ that should be actually passed at present from route constraints rule.

If $l p_{k}=l p_{\text {pre }}$, skip to Step 4 ; if $l p_{k}<l p_{\text {pre }}$, skip to Step 5 .

Step 3. Find logical control point of reading leak $l p_{\text {leak }}$ from route constraints rule based on $o_{k}$ and $l p_{k}$.

The system executes compensation reading from object $k$ and adds reading leak records

$\left(o_{k}, p_{\text {leak }}\right.$, "compensation," $\left.\tau\right)$ to the object information base.

Step 4. Add normal identification records to object information base.

Step 5. Abandon logical reading event $l p_{k}$, and skip to Step 1.

Algorithm 2: RFID complex event processing algorithm.

6.2. Production Planning and Scheduling Optimization Algorithm. For generalized Lagrangian relaxation algorithm used in this paper, planning and scheduling subproblems will be solved, respectively, in each time of Lagrangian iteration. Among each iteration, planning subproblem uses the sequencing results of scheduling in last iteration, and vice versa. That is, solving planning subproblem with fixed sequence (by accurate optimization method solved by branch and bound method) and solving scheduling by fixing the solved batch. Thus, this algorithm is essentially an alternating iteration method. Lasserre proposed to solve workshop production planning and scheduling integrated optimization problem by alternating iteration method, that is, alternating "solving planning problem with a fixed sequence" and "solving scheduling problem with fixed planning" $[32,36]$. It was proved that this algorithm can converge to a local optimal solution through limited iterations under the condition that planning and scheduling subproblems are solved by accurate optimization method. Solution to scheduling by fixed planning of this algorithm is actually to seek good scheduling with given planning, so that planning objectives can be further improved in the next iteration. It will be pointed out that "accurate optimization method applied to both planning and scheduling subproblems" is an ideal condition, as it is unpractical to solve scheduling subproblem by accurate method (Algorithm 3).

Comparing to Lasserre's method, this paper proposes to solve planning subproblem and scheduling subproblem in pairs in each time of iteration. These two subproblems are coordinated and optimized through Lagrangian multipliers.
In addition, generalized Lagrangian relaxation algorithm used in this paper is essentially a heuristic algorithm, and the end condition of it is set as the number of iterations reaches the prescribed number.

6.3. Numerical Simulations. Joint decision model is a nonconvex and nonconcave nonlinear programming model with a fraction. Traditional nonlinear programming approach can be adopted, such as GINO, gradient search, and semi-Newton method. However, these approaches tend to suffer from local minima. As the number of suppliers, manufacturers, and product categories increases, multiple components manufacturing lines will produce diverse products simultaneously and feed them to corresponding mixed-model assembly lines. The calculation and optimization solution becomes very complex and difficult. It is hard, if not impossible, for traditional nonlinear programming algorithms to solve the optimal solution.

This paper takes the automobile stamping workshop with five sets of equipment $\left(m_{1}, \ldots, m_{5}\right)$ as the case study example; the interval of production plan covers three production cycles. The normal production capacity of each cycle is 8hour working time (i.e., 28,800 s) and the largest overtime is $4,800 \mathrm{~s}$. Six kinds of workpieces can be stamped. The other relevant parameters are set as follows: $d_{i, 1}=650, d_{i, 2}=d_{i, 3}=$ 800; $R I_{i, t}=200 ; C p_{i, t}=C h_{i, t}^{+}=40, C h_{i, 1}^{-}=160, C h_{i, 2}^{-}=135$, $C h_{i, 3}^{-}=110, C b_{i, 1}^{-}=560, C b_{i, 2}^{-}=375, C b_{i, 3}^{-}=180, C u_{i, 1}^{-}=210$, $i=1, \ldots, 6, t=1, \ldots, 3$. Stamping processing capacity of each machine is assumed to be $m_{1}=m_{2}=650, m_{3}=m_{4}=850$, $m_{5}=1000$, respectively. In addition, the equipment with low 
Step 1 (initialize). Set coefficient $\lambda^{0}=0$ in formula (39), and select a group of $\bar{\beta}^{0}$ randomly. Set Lagrangian multiplier upgrade step size $\Delta \lambda$ and maximum number of iterations, iteration number $\eta=1$ and $V=+\infty$.

Step 2 (update penalty coefficient). Calculate new penalty coefficient, $\lambda^{\eta}=\lambda^{\eta-1}+\Delta \lambda$, and determine the value of $\theta^{\eta}$.

Step 3 (solve production planning subproblem). Obtain solution $\theta^{\eta}$ of $\bar{Z}_{2}\left(x \mid \theta^{\eta}, \bar{\beta}^{\eta-1}\right)$, where the solution of batch $X$ is denoted as $X^{\eta}$, and set $\bar{Q}^{\eta}=X^{\eta}$.

Step 4 (solve scheduling subproblem). Obtain the solution $\pi^{\eta}$ of $\bar{Z}_{3}\left(\pi \mid \theta^{\eta}, \bar{Q}^{\eta}\right)$, where solution of assignment variable $a$ is denoted as $a^{\eta}$, and set $\bar{\beta}^{\eta}=a^{\eta}, \bar{\delta}^{\eta}=\delta^{\eta}$.

Step 5 (target values of problems). Set target values $Z^{\eta}=\bar{Z}_{2}\left(x \mid \theta^{\eta}, \bar{\beta}^{\eta-1}\right)+\bar{Z}_{3}\left(\pi \mid \theta^{\eta}, \bar{Q}^{\eta}\right)$, sol $l^{T}=\left(x^{T}, \pi^{T}\right)$, and when $\eta=1$, set $U=s o l^{\eta}$.

Step 6 (determine whether the end condition is met). If $\eta$ is less than iterations, output target value $V$ and solution $U$, the algorithm ends; otherwise, set $\eta=\eta+1$, and skip to Step 2 .

Algorithm 3: Production planning and scheduling optimization algorithm.

TABLE 2

\begin{tabular}{lcccccc}
\hline Working procedure & $J_{1}$ & $J_{2}$ & $J_{3}$ & $J_{4}$ & $J_{5}$ & $J_{6}$ \\
\hline$m_{1}$ & 2 & 5 & 5 & 3 & 4 & 1 \\
$m_{2}$ & 2 & 5 & 3 & 1 & 2 & 3 \\
$m_{3}$ & 1 & 5 & 2 & 4 & 4 & 3 \\
$m_{4}$ & 5 & & 2 & 2 & 4 & 3 \\
$m_{5}$ & & & 1 & & & \\
\hline
\end{tabular}

stamping capacity cannot process those working procedures with high stamping requirement (see Table 2).

The production batch can be obtained from the above production plan model $X=\left(X_{1}, X_{2}, X_{3}\right)=(850,850$, $850,850,850,850)$, also $S I_{i}^{+}=S I_{i}^{-}=0(i=1,2,3)$. The shortage quantity of production in three cycles is initialized as $L_{1}=L_{2}=L_{3}=0$. The objective function of the production planning is achieved as 668300. If we assign the WIP to pass along the computed machining sequence, the completion time of each production cycle is beyond the maximally allowed processing time. It means that there is no match between production plan and production scheduling. However, if we adopt the model proposed in this paper to resolve the global optimum solution, the total completion times of the three production cycles are 33247, 33247, and 32567 , respectively. Each of them is less than its correspondingly maximally allowed production capacity. Therefore, the computed production plan is feasible, which indicates the effectiveness of the proposed optimization model.

\section{Conclusion}

Mixed-model assembly lines have been widely used in various industries, including automotive assembly, mobile phone making, and computer production. This paper introduces RFID technology to manufacturing workshop to convert manufacturing objects to be "smart manufacturing objects" which are enabled for dynamic interactions among them in real-time. Considering the theoretical model regarding the optimal configuration and framework integration of RFID sensor networks for general applications has not yet been well addressed, this paper proposes a novel generalized RFID-enabled MES model that integrates three submodels including RFID network configuration, the production planning, and scheduling optimization. This combined model overcomes the local minima defects of subobjective function perspective where linear summation approach is deployed for solving only one part of the overall problem. The individually formed subproblems, that include RFID optimal allocation, production planning and scheduling, could be solved by alternating iterations. The closely connected scheduling and production planning are well taken care of by setting the precomputed parameters as constraints. For production planning, to ensure its feasibility, the machine assignment and detailed scheduling parameters are treated as constraints when formulating production planning optimization model. To maintain the intercoordination, production planning subproblem and scheduling subproblem are solved in pairs in each time of iteration through Lagrangian multipliers. The optimization of such integrated model is complicated and belongs to NP-hard problem, which, however, could be solved using the proposed generalized Lagrangian decomposition approach that combines standard Lagrangian approach with Gauss-Seidel iterative principle. The algorithm analysis and numerical simulation have verified the feasibility and effectiveness of the proposed method for modeling and optimization.

In addition, reliable identification and tracking of manufacturing objects is the key to realize agile production management. Due to the influence of characteristics of wireless communication and complex application environment in manufacturing workshop, raw data acquired from RFID devices is often duplicated, missing, or with noise. By defining the RFID data flow as event, the real-time RFID data processing technology is proposed via construction of RFID event model which contains basic events and complex events. The proposed RFID configuration model could improve the accuracy of the MES monitoring information in real shop-floor environment. The proposed model structure and its optimization solution are quite generally applicable for 
converting the traditional MES into real-time interactive wireless manufacturing scenario.

\section{Notation}

K: $\quad$ Quantity of RFID devices for data acquisition

$\mathrm{INV}_{i}: \quad$ Initial inventory of product $i$ in the first production cycle

$J(m)$ : A set of working procedures that can be processed on machine $m$, excluding virtual ones

$J_{i}(m)$ : A set of working procedures that can be processed on machine $m$, includingprocesses of virtual product $J_{0}(i=1)$ and $J_{n+1}(i=2)$

$M(i, v)$ : A set of machines that can process $(i, v)$

$\mathrm{Ch}_{i, t}^{+}$: Unit penalty cost for safety stock excess of product $i$ in production cycle $t$

$\mathrm{Ch}_{i, t}^{-}$: Unit penalty cost for safety stock shortfall of product $i$ in production cycle $t$

$C p_{i, t}$ : Unit production expenses of product $i$ in production cycle $t$

$C b_{i, t}: \quad$ Unit shortage cost of product $i$ in production cycle $t$

$C u_{i, t}$ : Production preparation cost of product $i$ in production cycle $t$

$C s_{i, v}^{m}$ : Production adjustment costs (i.e., die change costs) of process $(i, v)$ on machine $m$

$C t_{m, t}$ : Unit overtime cost in production cycle $t$

$d_{i, t}: \quad$ Demands of product $i$ in cycle $t$

$M l_{i}$ : $\quad$ Upper limit of production lot of product $i$

$m_{i}: \quad$ Minimum lot size of product $i$

$\omega: \quad$ The scaling factor of overtime upper limit

$W_{t}$ : Available production capacity in production cycle $t$; here, it is assumed that each machine has the same production capacity

$X_{i, t}$ : Output of product $i$ in production cycle $t$

$L_{i, t}: \quad$ Shortage quantity of product $i$ in production cycle $t$

$y_{i, t}: \quad$ The value is 1 if product is being produced in production cycle $t$; otherwise it is 0

$B_{i, v}^{t}$ : $\quad$ Start time of process $(i, v)$ in production cycle $t$

$C_{i, v}^{t}: \quad$ Completion time of process $(i, v)$ in production cycle $t$

$F_{m, t}: \quad$ The completion time of the last processed workpiece on machine $m$ in cycle $t$

$\alpha_{i, v}^{m, t}: \quad$ The value is 1 if process $(i, v)$ is assigned to be processed on machine $m$ in cycle $t$; otherwise it is 0

$\delta_{i, v, j, w}^{m, t}$ : The value is 1 if process $(i, v)$ is in front of process $(j, w)$ on machine $m$ in cycle $t$; otherwise it is 0

$O_{m, t}$ : Overtime on machine $m$ in production cycle $t$.

\section{Conflict of Interests}

The authors declare that there is no conflict of interests regarding the publication of this paper.

\section{Acknowledgments}

The authors would like to thank the funding support by the University of Macau, Grant nos. MYRG153(Y1-L2)-FST11YZX and MYRG079(Y1-L2)-FST13-YZX. The authors would also like to thank the reviewers for their helpful and detailed comments.

\section{References}

[1] E. W. T. Ngai, K. K. L. Moon, F. J. Riggins, and C. Y. Yi, "RFID research: An academic literature review (1995-2005) and future research directions," International Journal of Production Economics, vol. 112, no. 2, pp. 510-520, 2008.

[2] A. Sarac, N. Absi, and S. Dauzre-Prs, "A literature review on the impact of RFID technologies on supply chain management," International Journal of Production Economics, vol. 128, no. 1, pp. 77-95, 2010.

[3] D. McFarlane and Y. Sheffi, "The impact of automatic identification on supply chain operations," The International Journal of Logistics Management, vol. 14, no. 1, pp. 1-17, 2003.

[4] F. Thiesse and E. Fleisch, "On the value of location information to lot scheduling in complex manufacturing processes," International Journal of Production Economics, vol. 112, no. 2, pp. 532547, 2008.

[5] R. G. Qiu, "RFID-enabled automation in support of factory integration," Robotics and Computer-Integrated Manufacturing, vol. 23, no. 6, pp. 677-683, 2007.

[6] D. McFarlane, S. Sarma, J. L. Chirn, C. Y. Wong, and K. Ashton, "Auto ID systems and intelligent manufacturing control," Engineering Applications of Artificial Intelligence, vol. 16, no. 4, pp. 365-376, 2003.

[7] P. Vrba, F. Macůrek, and V. Mařík, "Using radio frequency identification in agent-based control systems for industrial applications," Engineering Applications of Artificial Intelligence, vol. 21, no. 3, pp. 331-342, 2008.

[8] G. Q. Huang, Y. F. Zhang, and P. Y. Jiang, "RFID-based wireless manufacturing for walking-worker assembly islands with fixed-position layouts," Robotics and Computer-Integrated Manufacturing, vol. 23, no. 4, pp. 469-477, 2007.

[9] G. Q. Huang, Y. F. Zhang, and P. Y. Jiang, "RFID-based wireless manufacturing for real-time management of job shop WIP inventories," International Journal of Advanced Manufacturing Technology, vol. 36, no. 7-8, pp. 752-764, 2008.

[10] G. Q. Huang, Y. F. Zhang, X. Chen, and S. T. Newman, "RFIDenabled real-time wireless manufacturing for adaptive assembly planning and control," Journal of Intelligent Manufacturing, vol. 19, no. 6, pp. 701-713, 2008.

[11] S. Zhou, W. Ling, and Z. Peng, "An RFID-based remote monitoring system for enterprise internal production management," International Journal of Advanced Manufacturing Technology, vol. 33, no. 7-8, pp. 837-844, 2007.

[12] A. J. C. Trappey, T. H. Lu, and L. D. Fu, "Development of an intelligent agent system for collaborative mold production with RFID technology," Robotics and Computer-Integrated Manufacturing, vol. 25, no. 1, pp. 42-56, 2009. 
[13] C. Liu, L. S. Chen, and R. M. Romanowski, "An electronic material flow control system for improving production efficiency in integrated-circuit assembly industry," International Journal of Advanced Manufacturing Technology, vol. 42, no. 3-4, pp. 348$362,2009$.

[14] Z. X. Guo, W. K. Wong, S. Y. S. Leung, and J. T. Fan, "Intelligent production control decision support system for flexible assembly lines," Expert Systems with Applications, vol. 36, no. 3, pp. 4268-4277, 2009.

[15] R.-S. Chen and M. A. Tu, "Development of an agent-based system for manufacturing control and coordination with ontology and RFID technology," Expert Systems with Applications, vol. 36, no. 4, pp. 7581-7593, 2009.

[16] K. T. C. Poon, K. L. Choy, and H. C. W. Lau, "A RFID-based location tracking scheme for inbound operations in warehouse environment," in Proceedings of the Portland International Conference on Management of Engineering \& Technology (PICMET '08), pp. 872-877, July 2008.

[17] K. T. C. Poon, K. L. Choy, and H. C. W. Lau, "A real-time manufacturing risk management system: an integrated RFID approach," in Proceedings of the Portland International Center for Management of Engineering and Technology (PICMET '07), pp. 2872-2879, IEEE, August 2007.

[18] G. Huang, P. Wright, and S. T. Newman, "Wireless manufacturing: a literature review, recent developments, and case studies," International Journal of Computer Integrated Manufacturing, vol. 22, no. 7, pp. 579-594, 2009.

[19] G. Q. Huang, T. Qu, Y. Zhang, and H. D. Yang, "RFID-enabled product-service system for automotive part and accessory manufacturing alliances," International Journal of Production Research, vol. 50, no. 14, pp. 3821-3840, 2012.

[20] J. F. Benders, "Partitioning procedures for solving mixedvariables programming problems," Numerische Mathematik, vol. 4, no. 1, pp. 238-252, 1962.

[21] A. M. Geoffrion, "Generalized Benders decomposition," Journal of Optimization Theory and Applications, vol. 10, no. 4, pp. 237260, 1972.

[22] H. Everett III, "Generalized Lagrange multiplier method for solving problems of optimum allocation of resources," Operations Research, vol. 11, no. 3, pp. 399-417, 1963.

[23] M. L. Fisher, "The Lagrangian relaxation method for solving integer programming problems," Management Science, vol. 27, no. 1, pp. 1-18, 1981.

[24] M. Guignard and S. Kim, "Lagrangean decomposition: a model yielding stronger Lagrangean bounds," Mathematical Programming, vol. 39, no. 2, pp. 215-228, 1987.

[25] A. D. Dimitriadis, N. Shah, and C. C. Pantelides, "RTN-based rolling horizon algorithms for medium term scheduling of multipurpose plants," Computers and Chemical Engineering, vol. 21, no. 1, pp. S1061-S1066, 1997.

[26] H.-S. Yan, Q.-F. Xia, M.-R. Zhu, X.-L. Liu, and Z.-M. Guo, "Integrated production planning and scheduling on automobile assembly lines," IIE Transactions, vol. 35, no. 8, pp. 711-725, 2003.

[27] G. Sand and S. Engell, "Modeling and solving real-time scheduling problems by stochastic integer programming," Computers and Chemical Engineering, vol. 28, no. 6-7, pp. 1087-1103, 2004.

[28] J. D. Kelly and D. Zyngier, "Hierarchical decomposition heuristic for scheduling: coordinated reasoning for decentralized and distributed decision-making problems," Computers and Chemical Engineering, vol. 32, no. 11, pp. 2684-2705, 2008.
[29] M. Erdirik-Dogan and I. E. Grossmann, "Simultaneous planning and scheduling of single-stage multi-product continuous plants with parallel lines," Computers and Chemical Engineering, vol. 32, no. 11, pp. 2664-2683, 2008.

[30] H. Stefansson, N. Shah, and P. Jensson, "Multiscale planning and scheduling in the secondary pharmaceutical industry," AIChE Journal, vol. 52, no. 12, pp. 4133-4149, 2006.

[31] J. Kallrath, "Planning and scheduling in the process industry," OR Spectrum, vol. 24, no. 3, pp. 219-250, 2002.

[32] J.-B. Lasserre, "An integrated model for job-shop planning and scheduling," Management Science, vol. 38, no. 8, pp. 1201-1211, 1992.

[33] K. Haase and A. Kimms, "Lot sizing and scheduling with sequence-dependent setup costs and times and efficient rescheduling opportunities," International Journal of Production Economics, vol. 66, no. 2, pp. 159-169, 2000.

[34] I. Kacem, S. Hammadi, and P. Borne, "Approach by localization and multiobjective evolutionary optimization for flexible jobshop scheduling problems," IEEE Transactions on Systems, Man and Cybernetics C: Applications and Reviews, vol. 32, no. 1, pp. $1-13,2002$.

[35] I. Kacem, S. Hammadi, and P. Borne, "Pareto-optimality approach for flexible job-shop scheduling problems: hybridization of evolutionary algorithms and fuzzy logic," Mathematics and Computers in Simulation, vol. 60, no. 3-5, pp. 245-276, 2002.

[36] J. Lasserre, An Integrated Approach in Production Planning and Scheduling, vol. 411 of Lecture Notes in Economics and Mathematical Systems, Springer, Berlin, Germany, 1994. 


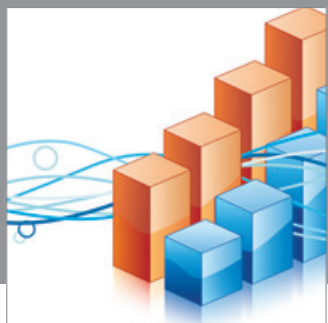

Advances in

Operations Research

mansans

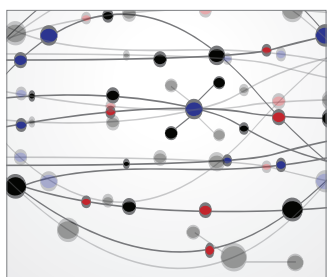

The Scientific World Journal
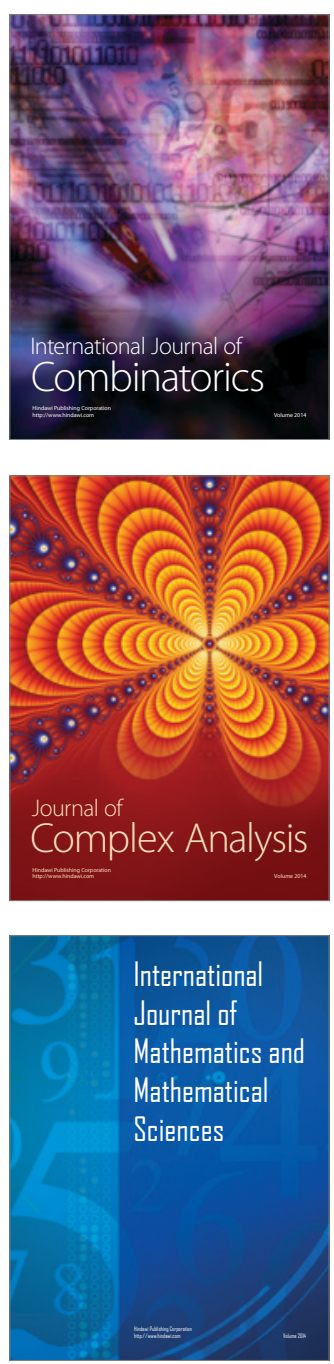
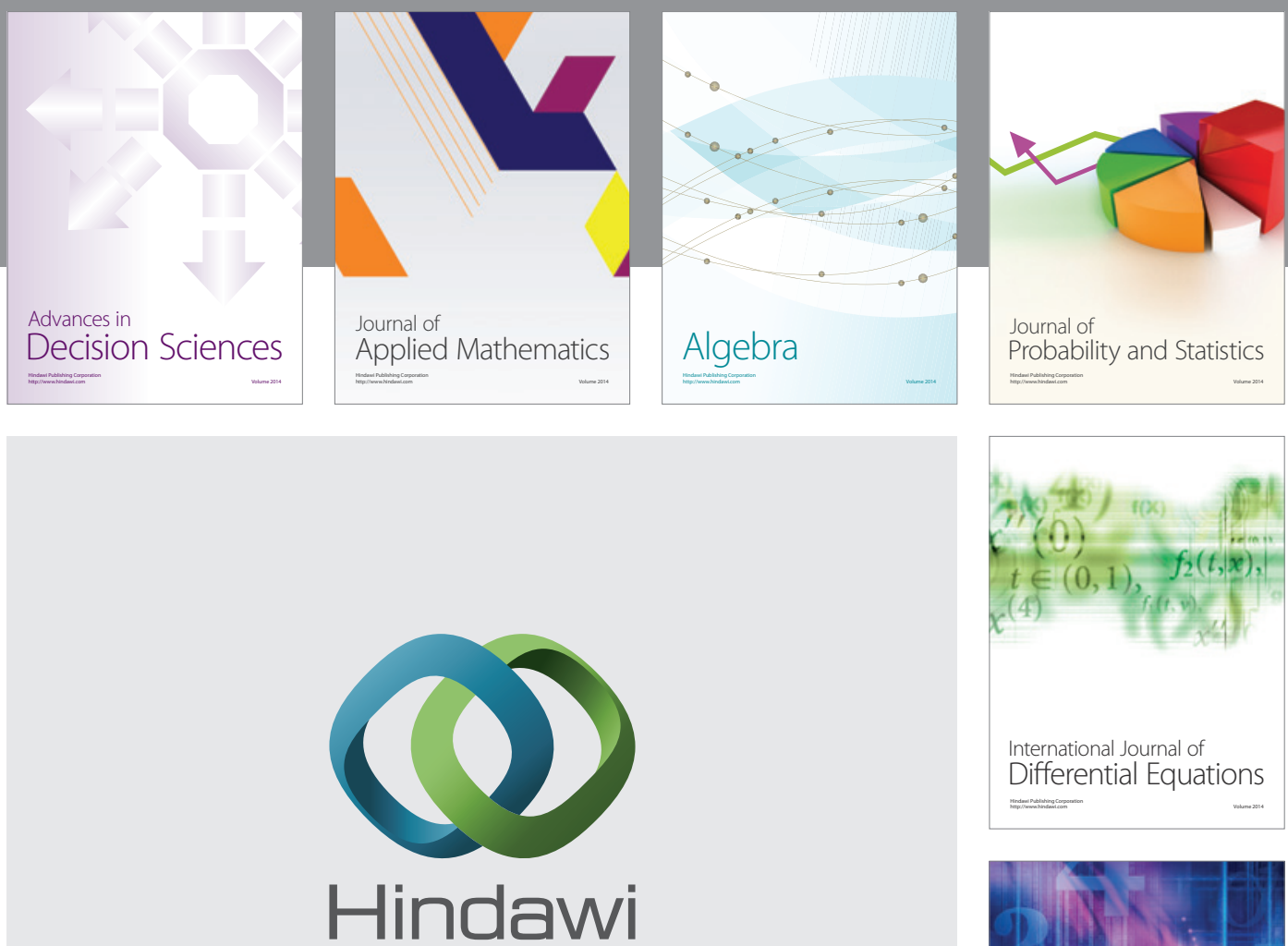

Submit your manuscripts at http://www.hindawi.com
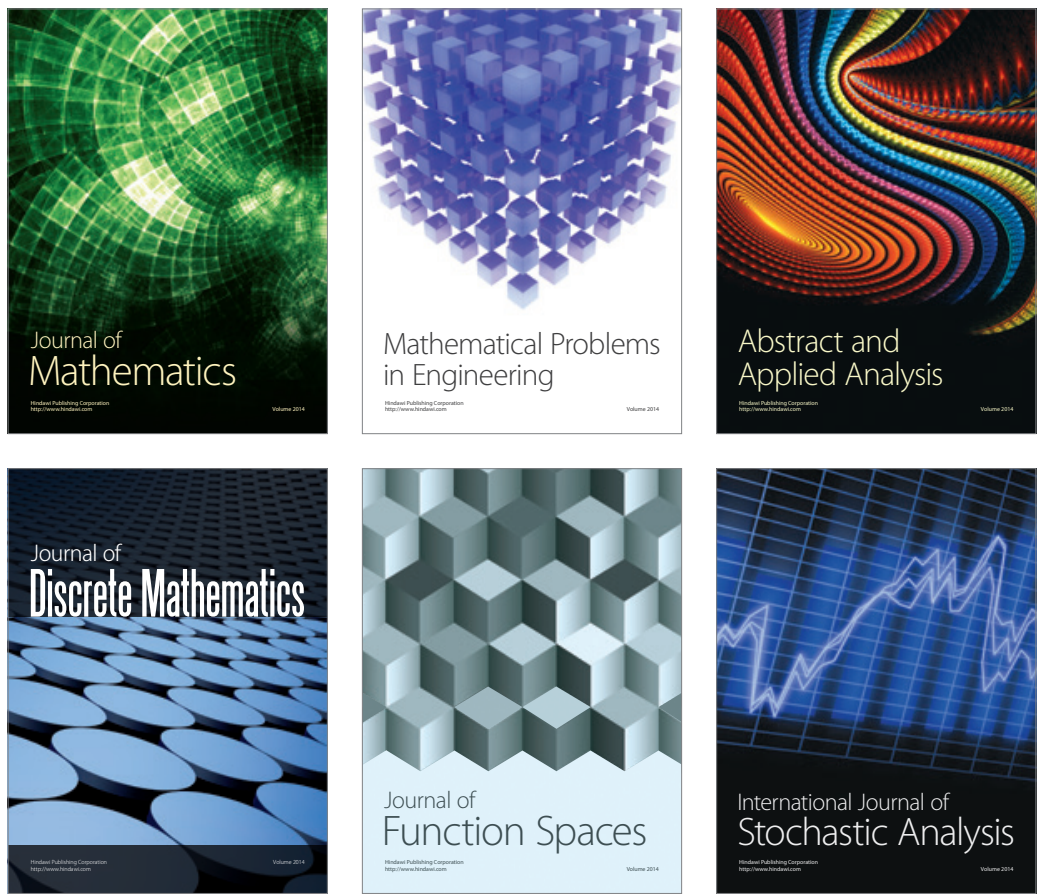

Journal of

Function Spaces

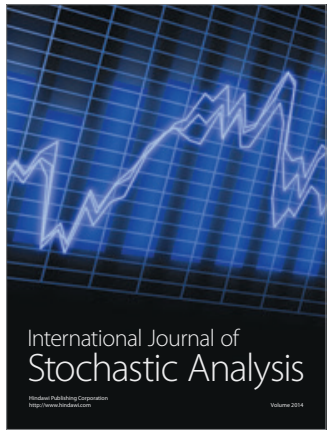

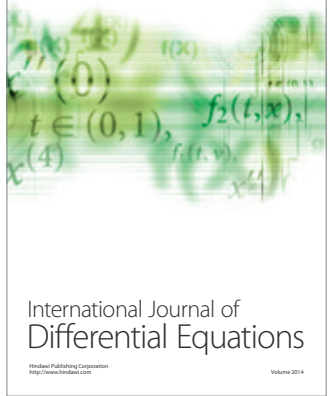
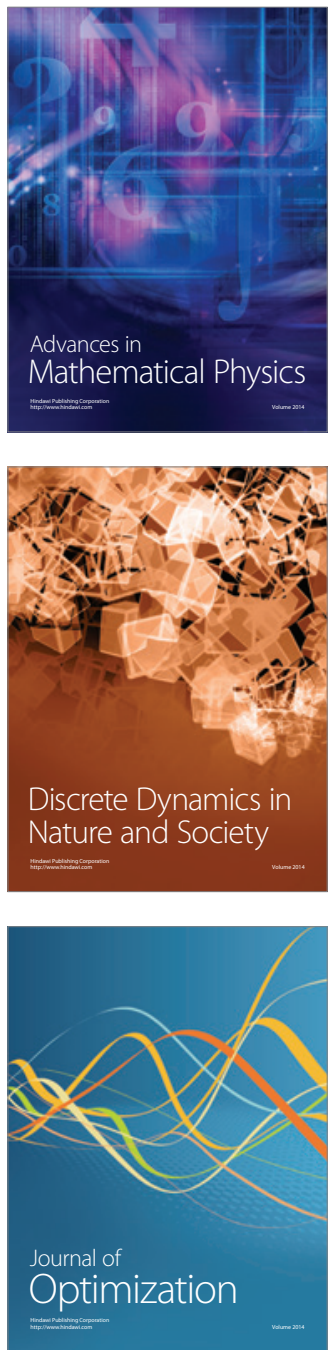